\title{
A model for intermediate quasi-molecular state and variants of chemical element synthesis
}

\author{
M. P. Kashchenko ${ }^{1,2,}$, V.F. Balakirev ${ }^{3}$ \\ ${ }^{1}$ Ural Federal University named after the first President of Russia B. N. Yeltsin, 19 Mira st., Yekaterinburg, 620002, Russia \\ ${ }^{2}$ Ural State Forest Engineering University, 37 Sybirskyi Tract st., Yekaterinburg, 620100, Russia \\ ${ }^{3}$ The Institute of Metallurgy Ural Branch of RAS, 101 Amundsen st., Yekaterinburg, 620016, Russia

$$
\text { †mpk46@mail.ru }
$$

In the present work, the simplest quasi-classic model of intermediate quasi-molecular state (IQS) is discussed. From the one hand, the model is based upon Bohr's idea about a binding electron orbit of hydrogen molecule having a round form with the orbital plane orthogonal to the section connecting the nuclei. From the other hand, the model permits coupling of electrons (with opposite spins) owing to non-potential contact interaction that had been introduced in hadronic mechanics of Santilli. In order to underline the specifics of the results of such interactions, term "isoparticles" is used in hadronic mechanics. Using a pair of bivalent oxygen ions, it is shown that the simplest model allows drawing the nuclei together until critical distances $R_{c} \approx 10^{-13} \mathrm{~m}$. In the discussion of the results, an additional possibility of element synthesis for IQS is noted. This possibility is connected with exchange of virtual isopositron-isoelectron pairs between the near-coming nuclei. The upper level of such interaction radius $\left[R_{\text {int }}\right]_{\max }$ corresponds to $R_{c} \approx 10^{-13} \mathrm{~m}$ in the case of exchange of virtual positroniums (with rest energy $\approx 1 \mathrm{MeV}$ ), while the lower level $\left[R_{\text {int }}\right]_{\min } \approx 10^{-15} \mathrm{~m}$ is realized in the case of virtual $\pi^{0}$-mesons interchange. As soon as in hadronic mechanics $\pi^{0}$-meson is interpreted as a bonded state of isopositron and isoelectron (with rest energy $\approx 135 \mathrm{MeV}$ ), the offered mechanism of exchange naturally allows the existence of virtual pairs with energies $\varepsilon$ in the interval $1 \mathrm{MeV}<\varepsilon<135 \mathrm{MeV}$. Therefore, by achievement of IQS, the channel for new elements synthesis opens and does not require decomposition of the initial nuclei till $\alpha$-particles with their following synthesis at opposing motion towards common center of attraction.

Keywords: quasi-molecular state model, hadronic mechanics, contact interaction, isoparticles, additional channel for elements synthesis.

УДК: 539.1 .01

\section{Модель промежуточного квазимолекулярного состояния} и варианты синтеза химических элементов

\author{
Кащенко М.П., ${ }^{1,2, \dagger}$, Балакирев В. Ф. ${ }^{3}$ \\ ${ }^{1}$ Уральский федеральный университет им. $1^{\text {го }}$ Президента России Б. Н. Ельцина, \\ ул. Мира, 19, Екатеринбург, 620002, Россия
}

${ }^{2}$ Уральский государственный лесотехнический университет, ул. Сибирский тракт, 37, Екатеринбург, 620100, Россия

${ }^{3}$ Институт металлургии Уральского отделения РАН, ул. Амундсена, 101, Екатеринбург, 620016, Россия

В данной работе рассматривается простейшая квазиклассическая модель промежуточного квазимолекулярного состояния (ПКС). С одной стороны, модель базируется на идее Бора о связующей электронной орбите молекулы водорода в форме окружности с плоскостью орбиты, ортогональной отрезку, соединяющему ядра. С другой стороны, в модели допускается спаривание электронов (с противоположными спинами) за счет непотенциального контактного взаимодействия, которое было введено в адронной механике Сантилли. Чтобы подчеркнуть специфику результатов подобных взаимодействий, в адронной механике используется термин изочастицы. На примере столкновения пары двухвалентных ионов кислорода показано, что простейшая модель ПКС допускает сближение ядер до критических расстояний $R_{c} \approx 10^{-13}$ м. При обсуждении результатов отмечается дополнительная для ПКС возможность синтеза элементов. Эта возможность заключается в обмене сближающихся ядер виртуальными изопозитрон-изоэлектронными парами. Верхняя граница радиуса такого взаимодействия $\left[R_{\text {int }}\right]_{\max }$ соответствует $R_{c}$ в случае обмена виртуальными позитрониями (с энергией покоя $\approx 1 \mathrm{MэB),} \mathrm{тогда} \mathrm{как} \mathrm{нижняя} \mathrm{граница}\left[R_{\text {int }}\right]_{\text {min }} \approx 10^{-15}$ м реализуется при обмене виртуальными $\pi^{0}$-мезонами. Поскольку в адронной механике $\pi^{0}$-мезон интерпретируется как связанное состояние изопозитрона и изоэлектрона (с энергией покоя $\approx 135$ МэВ), то предлагаемый механизм обмена естественно допуска- 
ет существование виртуальных пар с энергиями $\varepsilon$ из интервала 1 МэВ $<\varepsilon<135$ МэВ. Следовательно при достижении ПКС открывается канал для синтеза новых элементов, не требующий распада исходных ядер на $\alpha$-частицы с последующим их синтезом при встречном движении к общему центру притяжения.

Ключевые слова: модель квазимолекулярного состояния, адронная механика, контактное взаимодействие, изочастицы, дополнительный канал синтеза элементов.

\section{1. Введение}

В настоящее время реализация низкоэнергетических ядерных реакций является установленным фактом. Причем основное внимание сосредоточено на синтезе при реакциях легких ядер, что обусловлено возможностью создания эффективных источников энергии. Как правило, руководящая идея состоит в необходимости сближения ядер до критического расстояния $R_{c} \sim 10^{-13} \mathrm{M}$, при котором начинается эффективное туннелирование легких ядер [1]. В связи с этим в качестве одного из способов сближения ядер стало использование твердотельной матрицы, насыщаемой легкими элементами. Собственно, уже в первых экспериментах Флейшмана и Понса [2], подобный синтез начинался лишь после предварительного долгого (66 суток) насыщения палладиевого электрода дейтерием. Успешно используется порошок титана, насыщенный дейтерием, для синтеза трития [3]. Убедительный теоретический анализ [4] показывает, что сближение ядер в твердой матрице, увеличивающее прозрачность барьера при туннелировании, достигается при учете нелинейных колебаний.

Однако наряду с холодным синтезом легких элементов имеются достоверные данные о низкоэнергетическом синтезе элементов со средними и большими значениями масс в расплавах, растворах и даже дистиллированной воде (см., например, [5-7]). Так, эксперименты по обработке воды электрическим током (глава 4 в [6]) демонстрируют, что в значительном количестве образуются не только углерод, но и элементы (например, $\mathrm{Si}$ и $\mathrm{Fe}$ ) с зарядовыми числами $Z$ ядер, значительно превышающими $Z=8$ для атомов кислорода. Анализ экспериментальных данных для двух различных электролизеров [8] показал, что ионы кислорода, которые естественно рассматривать в качестве исходных структурных элементов, имеют энергии $\leq 1$ кэВ. В связи с этим в [8], высказана гипотеза о возникновении в процессе столкновения ионов (атомов) промежуточных квазимолекулярных состояний (ПКС), способных обеспечить сближение ядер до критического расстояния $R_{c} \sim 10^{-13} \mathrm{M}$, достаточного для начала туннелирования $\alpha$-частиц, ведущего, в первую очередь, к синтезу элементов с $\alpha$-кластеризованными ядрами. Кроме того, в [8] отмечено, что идея о существовании ПКС имеет более общее физическое значение.

При построении модели ПКС естественно полагать, что сближение ядер возможно лишь благодаря увеличению электронной плотности в межъядерном пространстве, экранирующей процесс отталкивания ядер и обеспечивающей их сближение за счет притяжения к общему отрицательному заряду. Целесообразно начать с некоторого простейшего варианта, позволяющего выполнить оценки порядков величин, допускающего легкую проверку расчетов и стимулирующего конструктивный критический анализ.

В качестве исходных примем следующие позиции. Во-первых, будем рассматривать задачу о столкновении одинаковых атомов (ионов) с равными зарядами ядер $q_{1}=q_{2}=q$, образующих в обычных условиях молекулу с расстоянием $2 d_{0}$ между ядрамя. Напомним, в схеме трех точечных зарядов, лежащих на одной прямой, при помещении в центр отрезка длиной $2 d$ отрицательного заряда $q_{3}=-q / 4$ заряды находятся в состоянии неустойчивого равновесия (при любой величине $d$ ). Разумеется, в реальности речь должна идти о некоторых динамических состояниях электронов, обеспечивающих высокую вероятность обнаружения отрицательного заряда в межъядерном пространстве и стабилизирующих размер $2 d<2 d_{0}$.

Во-вторых, используем наглядное квазиклассическое описание ПКС, допускающее орбитальное (по окружности) движение электронов при условии квантования момента импульса. К вопросу о правомерности использования траекторий, которое, как известно, выходит за рамки ортодоксальной квантовой механики, мы вернемся при обсуждении результатов.

В-третьих, из различных модельных конфигураций орбит для движущихся электронов ограничимся орбитами, расположенными в плоскости, ортогональной отрезку, соединяющему ядра с положением общего центра орбит в середине отрезка.

В-четвертых, будем полагать, что в процессе столкновения атомов возникает контактное (непотенциальное) взаимодействие электронов, при котором за счет перекрытия их волновых пакетов (на адронных масштабах $\sim 10^{-15}$ м) обеспечивается притяжение электронов с противоположными спинами. Теория этого взаимодействия была развита в адронной механике Сантилли (см., например, [9]), представляющей глубокое обобщение квантовой механики. Появление нового качества у частиц в таком состоянии отражается введением термина - «изочастица».

В-пятых, четность общего числа электронов позволяет использовать для оценки величины $d$ простейшую модель, в которой все электроны связаны в изоэлектронные пары (с противоположными спинами), подчиняются статистике Бозе-Эйнштейна и располагаются на единственной круговой орбите. Уместно отметить, что модель подобной орбиты для пары обычных электронов, обеспечивающих связь протонов в молекуле водорода, впервые предложил Бор [10].

Таким образом, цель работы показать, что существует, как минимум, одна модель ПКС, позволяющая обеспечить сближение ядер атомов на расстояние $2 d \sim R_{c} \sim 10^{-13}$ м и обсудить перспективы развития и использования модели. 


\section{2. Оценка расстояния между ядрами в простейшей модели ПКС}

На Рис. 1 представлена простейшая модель ПКС в виде единственного кольцевого электронного тока с радиусом $R$ и плоскостью кольца, ортогональной линии, соединяющей пару ядер с равными зарядами $+q=Z_{1} e_{+}$ $\left(Z_{1}-\right.$ зарядовое число ядра элемента, $e_{+}$- элементарный положительный заряд). На кольцевой орбите электроны присутствуют в виде связанных пар с противоположными спинами. На рисунке схематично представлены две такие пары (противоположные стрелки внутри малой окружности), находящиеся в электрическом поле ядер, причем символом $E_{\perp}$ обозначена напряженность результирующего электрического поля, пропорциональная центростремительной силе, удерживающей связанные пары на орбите. На ядра действует сила притяжения в направлении напряженности электрического поля $E$, создаваемого электронами на оси кольца, и сила взаимного отталкивания в направлении $E_{+}$. Если сталкиваются нейтральные атомы, то легко убедиться, что величина $E$ в точках, отстоящих на расстояние х от плоскости кольца, принимает значения

$$
E_{-}=-q x /\left[2 \pi \varepsilon_{0}\left(R^{2}+x^{2}\right)^{3 / 2}\right] .
$$

Условие электростатического равновесия для положения ядер (при $x= \pm d$ )

$$
\left|E_{-}\right|=q d /\left[2 \pi \varepsilon_{0}\left(R^{2}+d^{2}\right)^{3 / 2}\right]=E_{+}=q /\left[4 \pi \varepsilon_{0}(2 d)^{2}\right]
$$

дает связь между $R$ и $d$ :

$$
R=\sqrt{3} d
$$

В случае столкновения отрицательно заряженных ионов соотношение (3) модифицируется

$$
R=d\left\{\left[4 Z_{2} / Z_{1}\right]^{2 / 3}-1\right\}^{1 / 2}, \quad Z_{2}=2\left(Z_{1}+\mathrm{n}\right),
$$

где $Z_{2}$ - число электронов на кольцевой орбите, $n-$ валентность иона (например, для $\mathrm{O}^{2-} n=2$ и $Z_{2}=20$ ). Очевидно, что при $Z_{2}=2 Z_{1}$, (4) переходит в (3).

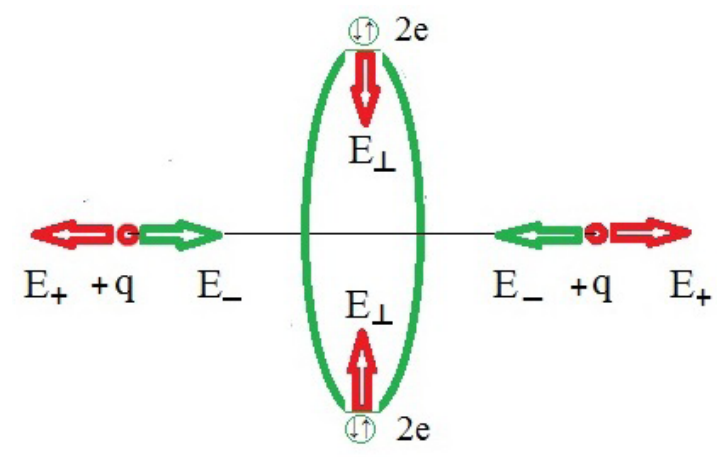

$2 \mathrm{R}$

$2 d$

Рис. 1. Схема простейшей модели ПКС в состоянии электростатического равновесия (отношение $R / d$ уменьшено по сравнению с расчетным значением).

Fig. 1. The scheme of the simplest IQS model in the state of electrostatic equilibrium (the $R / d$ ratio is reduced in comparison with the calculated value).
Напряженность поля на круговой орбите, создаваемая зарядами двух ядер, дается соотношением

$$
E_{\perp+}=3 \sqrt{3} q /\left(16 \pi \varepsilon_{0} R^{2}\right)
$$

где учтено равенство (3). При выполнении (4)

$$
E_{\perp+}=\left(Z_{1}^{2} e / 8 \pi Z_{2} \varepsilon_{0} R^{2}\right)\left\{\left[4 Z_{2} / Z_{1}\right]^{2 / 3}-1\right\}^{3 / 2} .
$$

При $Z_{2}=20, Z_{1}=16$ из (6) следует

$$
E_{\perp+} \approx 11.119 e /\left(4 \pi \varepsilon_{0} R^{2}\right) .
$$

Кроме напряженности (7) следует учесть вклад отталкивания электронов. Полагаем, что на круговой орбите симметрично располагаются 10 пар связанных изоэлектронов с массами $m_{e}^{*}$ (в общем случае $m_{e}^{*}$ не равна массе свободного электрона $\left.m_{e}\right)$. Считаем, что на каждую из локализованных пар с зарядом $2 e$ действует суперпозиционная кулоновская сила от девяти пар (плоский угол $\alpha$ между радиусами векторами соседних пар составляет $36^{\circ}$ ). Тогда получаем

$$
\begin{gathered}
E_{\perp-} \approx\left[e /\left(4 \pi \varepsilon_{0} R^{2}\right)\right]\left\{[\sin (\alpha / 2)]^{-1}+[\sin \alpha]^{-1}+\right. \\
\left.+[\sin (3 \alpha / 2)]^{-1}+[\sin 2 \alpha]^{-1}+0.5\right\} \approx 7.725 e /\left(4 \pi \varepsilon_{0} R^{2}\right) .
\end{gathered}
$$

Полагаем $m^{*}=Z_{2} m_{e}^{*} \equiv Z_{2}\left(m_{e}^{*} / m_{e}\right) m_{e}\left(m_{e} \approx 9.1 \cdot 10^{-31}\right.$ кг $)$. На каждый из $Z_{2}$ элементарных зарядов $-e\left(e \approx 1.6 \cdot 10^{-19} \mathrm{~K} л\right)$ действует сила, которую рассматриваем в качестве центростремительной

$$
e E_{\perp}=m_{e}^{*} v^{2} / R, \quad E_{\perp} \approx 3.394 e /\left(4 \pi \varepsilon_{0} R^{2}\right),
$$

где $v-$ орбитальная скорость электронов. Кроме того, налагаем условие квантования момента импульса

$$
m^{*} v R=\hbar k
$$

где $\hbar \approx 1,054 \cdot 10^{-34}$ Дж·с, а $k$ - целое число.

Заметим, что в рассматриваемом примере столкновения ионов $\mathrm{O}^{2-}$ исходным основным состояниям ионов соответствуют полностью заполненные электронные оболочки (как у неона), имеющие нулевые суммарные орбитальные и спиновые моменты. Однако из-за предшествующих финальному взаимодействию нецентральных столкновений ионы могут иметь ненулевые моменты импульса, так что появление конечного момента импульса, связанного с орбитальным движением электронных пар, не противоречит закону сохранения момента импульса. В связи с этим величину $k$ естественно рассматривать как феноменологический параметр, требуя, например, совпадения $R$ с величиной $R_{c} \sim 10^{-13} \mathrm{M}$.

Из (9), (10) находим

$$
\begin{aligned}
& R \approx R_{B} k^{2} /\left(3.4\left(m_{e}^{*} / m_{e}\right) Z_{2}^{2}\right), \quad R_{B}=4 \pi \varepsilon_{0} \hbar^{2} /\left(m_{e} e^{2}\right), \\
& v=\hbar k /\left(Z_{2} m_{e}^{*} R\right) \approx 3.4\left(m_{e}^{*} / m_{e}\right) \hbar Z_{2}^{2} /\left(Z_{2} m_{e}^{*} k R_{B}\right)= \\
&=3.4 Z_{2}^{2} \hbar /\left(m_{e} R_{B} k\right),
\end{aligned}
$$

где $R_{B}$ - радиус первой орбиты атома водорода в модели Бора. Примем $\left(m_{e}^{*} / m_{e}\right)=1$ и подставим в (11) и (12) $Z_{2}=20, R_{B} \approx 5 \cdot 10^{-11}$ м. Тогда интересующим нас значениям $R \sim R_{c} \sim 10^{-13}$ м соответствует $k=2$, при котором $R \approx 1.47 \cdot 10^{-13} \mathrm{M} \approx 3 \cdot 10^{-3} R_{B}$, а скорость $v \approx 0.793 \cdot 10^{8} \mathrm{M} / \mathrm{c} \approx 0.264 c$. Поскольку $v$ порядка скорости 
света $c$, то, строго говоря, следует учитывать релятивистские поправки, однако, при оценках порядков величин, это не имеет принципиального значения, так как множитель $\left(1-(\mathrm{v} / \mathrm{c})^{2}\right)^{1 / 2} \approx 0.964$ еще близок к 1 .

Заметим, что движению зарядов по круговой орбите сответствует сила тока $I$ и магнитная индуция в центре орбиты $B$ :

$$
I=Z_{2} e v / 2 \pi R, \quad B=\mu_{0} I / 2 \pi R,
$$

где $\mu_{0}=4 \pi \cdot 10^{-7}$ Нн/м - магнитная постоянная. При $Z_{2}=20$, $R \approx 1.47 \cdot 10^{-13} \mathrm{M}, v \approx 0.793 \cdot 10^{8} \mathrm{M} / \mathrm{c}, e=1.6 \cdot 10^{-19}$ Кл имеем $I \approx 275 \mathrm{~A}, B \approx 3.74 \cdot 10^{8}$ Тл.

Для ионов кислорода при $Z_{1}=8$ и $Z_{2}=20$ значение $\left\{\left[4 Z_{2} / Z_{1}\right]^{2 / 3}-1\right\}^{1 / 2} \approx 1.9083$. Тогда, согласно (4), $R \approx 1.91 d$, и расстояние $2 d \approx 1.54 \cdot 10^{-13} \mathrm{M} \sim R_{c} \sim 10^{-13} \mathrm{M}$.

\section{3. Обсуждение результатов}

\section{1. О контактном взаимодействии в адронной механике}

Прежде всего, констатируем, что в предложенной модели ПКС оказывается возможным сближение ядер до расстояний, меньших на два порядка боровского радиуса. Более того, при $\left(m_{e}^{*} / m_{e}\right)>1$ расстояние между ядрами, согласно (11), может еще уменьшиться. Очевидно, что при построении модели ПКС центральную роль выполняет допущение о возможности спаривания электронов за счет контактного взаимодействия. Причем формирование единой орбиты для пар изоэлектронов можно рассматривать как бозе-конденсацию в общем состоянии пар изоэлектронов, возникающих в процессе перекрытия электронных оболочек. Ясно, что модель ПКС относится к временной перестройке всей электронной структуры, а не только внешних оболочек ионов.

Уместно отметить, что термин «контактное взаимодействие» вводится и в квантовой механике атома: для сферически симметричных $s$-состояний радиальная часть волновой функции максимальна в центре ядра, то есть при нулевом радиусе взаимодействия (например, в центре протона для атома водорода). Однако линейность, обратимость и локальность квантовой механики не позволяют рассматривать нейтрон, как связанное состояние протона и электрона. В адронной же механике трактовка нейтрона, в качестве связанного состояния изопротона и изонейтрона, является основой одного из вариантов получения чистой энергии. Характерно, что масса связанных изочастиц, из-за непотенциальной природы контактного взаимодействия, превышает массу свободных частиц. В частности, масса покоя нейтрона превышает сумму масс свободных протона и электрона.

\section{2. О правомерности использования орбит электронов}

Обсудим теперь вопрос о правомерности использования траекторий применительно к движению микрообъектов. Прежде всего, напомним, что отказ от жесткого детерминизма в квантовой механике опирается на соотношения неопределенностей для координат и им- пульсов, а также на интерпретацию волновых свойств частиц. Тем не менее, для частиц с достаточно высокими энергиями и импульсами (а значит, малыми длинами волн де Бройля $\lambda$ ) допускается использование траекторий (например, электронных орбит в ридберговских атомах). При такой интерпретации радиусы орбит $R$ должны быть заведомо больше $\lambda$. В интересующем нас случае $R \sim 10^{-13}$ м это, в принципе, возможно за счет релятивистской коррекции импульса.

Имеется, однако, и альтернативная возможность. Как показал Бом [11], процесс дифракции электронов на двух щелях, считающийся одним из традиционных подтверждений вероятностной интерпретации квантовой механики, допускает описание с использованием индивидуальных траекторий электронов, но при введении нелокального квантового потенциала. Нелокальность потенциала подразумевает нарушение ограниченности скорости распространения взаимодействия величиной $c$. Напомним также о парадоксе Эйнштейна-Подольского-Розена [12], который при анализе мысленного эксперимента для запутанных состояний пары микрочастиц приводит к выводу о нарушении принципа близкодействия в квантовой механике. На наличие нелокальности указывает, по-видимому, эксперимент с запутанными состояниями микрочастиц, поставленный для проверки выводов [12] (см. обсуждение в [13]). Не исключено, что выход из этой коллизии кроется в развитии представлений о природе пространства-времени (см., например, [14-15]).

\section{3. О модели с парой круговых орбит}

Менее принципиальный вопрос касается использования единственной круговой траектории. Ясно, например, что порядок величины при оценке $d$ сохранится и в случае двух концентрических кольцевых орбит для связанных пар изоэлектронов. Причем допустим вариант центрального столкновения одинаковых ионов, не имеющих моментов импульса. Действительно, не нарушая закон сохранения момента импульса, можно полагать, что суммарные моменты импульсов изоэлектронов на каждой из орбит имеют равные значения, но противоположные направления. Разумеется, для орбит (особенно с меньшим радиусом и меньшим числом изоэлектронных пар) может потребоваться релятивистская коррекция оценок.

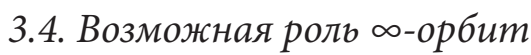

Касаясь построения других моделей ПКС, отметим, что представляет интерес исследование ळ-орбит (в форме восьмерки, охватывающей ядра), как в роли самостоятельного варианта, так и в комбинации с круговой орбитой (орбитами) типа рассмотренной выше. Заметим, что $\infty$-орбита, в качестве связующей для молекулы водорода, анализировалась Сантилли (модель «изоэлектрониума»), и было установлено притяжение для пары изоэлектронов с противоположными спинами при синхронном прохождении межъядерной области. При комбинированном варианте и сохранении симме- 
тричной круговой орбиты плоскость $\infty$-орбиты естественно считать ортогональной плоскости круговой орбиты. Этот выбор отражен на Рис. 2, где представлено состояние, когда пара связанных изоэлектронов уже находится в общем центре орбит, ядра сближаются за счет преобладания притяжения над отталкиванием $\left(\left|E_{-}\right|>E_{+}\right)$, но расстояние $d$ еще порядка $R_{B} / Z_{1}$.

Перспективность комбинированной модели, на наш взгляд, заключается в возможности захвата изоэлектронной пары и ее локализации (с точностью до «нулевых колебаний») в центре кольцевого тока. При такой конфигурации ПКС для сталкивающихся ионов $\mathrm{O}^{2-}$ легко показать, что выполнению условия $\left|E_{-}\right|=E_{+}$удовлетворяет соотношение

$$
R=d[2(3 \sqrt[3]{3}-1)]^{1 / 2} \approx 2.6 d
$$

то есть, по сравнению с чисто кольцевой конфигурацией (для которой, согласно $(4), R \approx 1.91 d)$, имеет место дополнительное сближение ядер.

Если локализации пары изоэлектронов в центре кольцевой орбиты не происходит, но имеет место существенное замедление пары (вследствие формирования массы $m^{*}$ ) при прохождении межъядерной области, то возможна периодическая модуляция межъядерного расстояния.

\section{5. О дополнительном канале синтеза элементов}

Наиболее ярким примером следствий адронной механики является трактовка $\pi^{0}$-мезона как связанного состояния изопротона и изопозитрона. Напомним, энергия покоя $\pi^{0}$-мезона составляет $\approx 135$ МэВ, то-

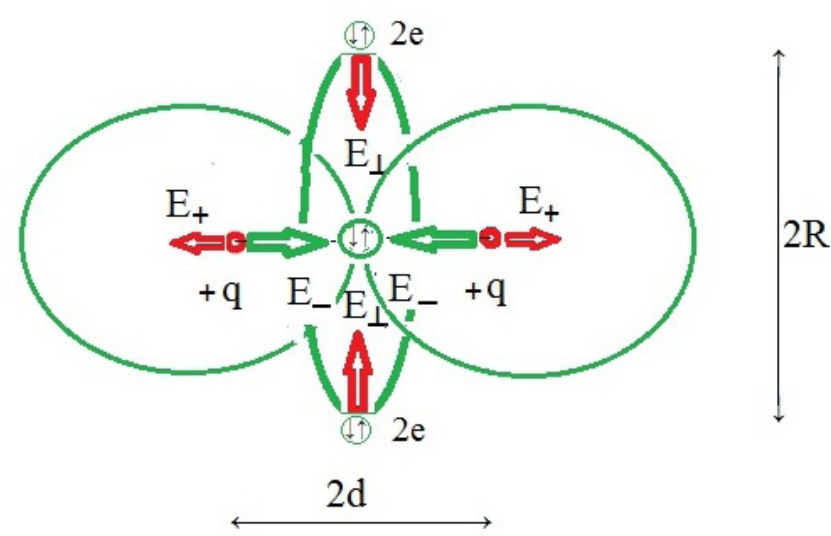

Рис. 2. Схема взаимного расположения орбит в комбинированной модели формирования ПКС.

Fig. 2. The scheme of the mutual arrangement of orbits in the combined model for the IQS formation. гда как сумма энергий покоя электрона и позитрона $\approx 1$ МэВ. В связи с этим естественно допустить, что возможно возникновение виртуальных изоэлектрон-изопозитронных пар, имеющих спектр энергий из интервала $1 \mathrm{Mэ} \mathrm{B} \leq \Delta E \leq 135 \mathrm{MэВ,} \mathrm{спектр} \mathrm{времен} \mathrm{суще-}$ ствования $\Delta \tau \sim \hbar / 2 \Delta E$ и интервал радиусов взаимодействия $R_{\text {int }} \sim c \Delta \tau \sim c \hbar / 2 \Delta E$, то есть

$$
10^{-13} \mathrm{M} \geq R_{\text {int }} \geq 7 \cdot 10^{-16} \mathrm{M} \text {. }
$$

Из (15) следует, что сближение ядер до расстояний $\sim 10^{-13}$ м в состоянии ПКС открывает канал для синтеза химических элементов за счет прямого обмена исходных ядер виртуальными изоэлектрон-изопозитронными парами (вплоть до $\pi^{0}$-мезонов), не требующего распада исходных ядер на $а$-частицы с последующим их синтезом при встречном движении к общему центру притяжения.

В связи с указанным механизмом возникает возможность синтеза элементов в реакциях с удвоениями массовых чисел при столкновениях предварительно синтезированных элементов. В частности, один из вариантов синтеза железа, наблюдаемого при электролизе воды [6], естественно сопоставить с процессом столкновения двух ионов кремния (с двумя последующими $\beta$-распадами [8]), полагая, что кремний предварительно синтезируется при столкновениях пар ионов кислорода.

\section{4. Заключение}

Проведенное рассмотрение показало, что факты низкотемпературного синтеза новых элементов можно связать с формированием промежуточных квазимолекулярных состояний, возникающих при глубоко неупругом столкновении отрицательных ионов (с сохранением коллектива электронов) и обеспечивающих притяжение ядер к избыточной электронной плотности в межъядерной области ПКС. Наиболее вероятным механизмом неупругого взаимодействия представляется контактное взаимодействие электронов, ведущее к их спариванию с изменением статистических свойств (бозе-частицы с нулевым спином).

Сближение ядер до расстояний $\sim 10^{-13}$ м при возникновении ПКС не только обеспечивает возможность встречного туннелирования легких ядер (дейтерия, трития, гелия), но и открывает канал синтеза ядер (и соответствующих химических элементов) за счет обмена виртуальными изоэлектрон-изопозитронными парами. Очевидно, что заслуживают внимания оба сценария.

Подчеркнем - полученные выводы не выходят за рамки уже существующих физических представлений, расширяя, тем не менее, арсенал привычной методологии применительно к синтезу элементов. 


\section{Литература/References}

1. S.S. Gershtein, Yu. V. Petrov, L.I. Ponomarev. Sov. Phys. Usp. 33(8), 591 (1990). DOI: 10.1070/PU1990v033n08ABEH002619

2. M. Fleischmann, S. Pons. J. Electroanal., 261, 301 (1989).

3. D.D. Afonichev, T.I. Nazarova. Letters on materials. 7(1), 17 (2017). (in Russian) [Д.Д. Афоничев, Т. И. Назарова. Письма о материалах. 7(1), 17 (2017).] DOI: $10.22226 / 2410-3535-2017-1-17-20$

4. V.I. Dubinko, D. V. Laptev. Letters on materials. 6(1), 16 (2016). DOI: 10.22226/2410-3535-2016-1-16-21

5. V.V. Krymskiy, and V.F. Balakirev. Doklady Physical Chemistry. 385(4-6), 197 (2002). DOI: 10.1023/A:1019919424464

6. V.F. Balakirev, V.V. Krymskiy, B.V. Bolotov et al. Interconversion of chemical elements. Ekaterinburg, UB RAS (2003) 97 p. (in Russian) [В.Ф. Балакирев, В. Крымский, Б. В. Болотов и др. Взаимопревращения химических элементов. Екатеринбург, УрО РАН (2003) 97 c.]

7. V.V. Krymskiy, V.F. Balakirev, N.V. Plotnikova. J. Chem. Chem. Eng. 9, 211 (2015). DOI: 10.1265/19347375/2015.03.007

8. M. P. Kashchenko, V. F. Balakirev. Letters on materials. 7(4),
380 (2017). DOI: 10.22226/2410-3535-2017-4-380-383

9. R. M. Santilli. Foundations of Hadronic Chemistry. With Applications to New Clean Energies and Fuels. BostonDordrecht-London, Kluwer Academic Publishers (2001) 554p.

10. N. Bohr. In: Phil. Mag. 26(151), 1-25; 26(153), 476-502, 857-875 (1913). [Бор Н. В книге: Избранные научные труды. Т. 1. (Под ред. Е.И. Тамма, В.А. Фока, Б.Г. Кузнецова. Составитель У.И. Франкфурт). Москва, Наука (1970) 676 с. О строении атомов и молекул. с. $84-148$.

11. D. Bohm. Phys. Rev. 85, 166 (1952).

12. A. Einstein, B. Podolsky and N. Rosen. Phys. Rev. 47, 777 (1935).

13. G. Greenstein, A.G. Zajonc. The Quantum Challenge. Modern Research on the Foundatios of Quantum Mechanics. Boston-Toronto-London-Singapore, Jones and Bartlett Publishers (2006) 296p.

14. R. O. Bartini. Soviet Physics. Doclady. 163(4), 861 (1965). (in Russian) [Р.О. Бартини. ДАН СССР. 163(4), 861 (1965).]

15. V.I. Kostitsyn. Theory of multidimensional spaces. Moscow, Book house "LIBROCOM" (2013) 136p. (in Russian) [В.И. Костицын. Теория многомерных пространств. Изд. 3-е, доп. Москва, Книжный дом “ЛИБРОКОМ" (2013) 136с.] 